\title{
A Deep Learning Enabled Smart Shopping Cart
}

\author{
Lakshmi Narayanan', Dhanya Sudhakaran², Subhika \\ Grandhe ${ }^{3}$, Namra lqbal ${ }^{4}$ and Jimcymol James*5 \\ 1,2,3,4 Manipal Academy of Higher Education, Dubai Campus, Dubai, U.A.E. \\ ${ }^{5}$ Manipal Academy of Higher Education, Dubai Campus, Dubai, U.A.E.
}

\section{ABSTRACT}

Shopping malls and supermarkets are equipped with shopping trolleys to ease the shopping activity. Various literature and models are existing for a smart shopping cart. However, there is no solution or research made to address the problem of abandoned trolleys. The main motive of the paper is on tackling the abandoned trolley problem as the environmental concerns and accidents caused to pedestrians and road users need attention just as much as implementing artificial intelligence and internet of things (IoT) principles to deliver intelligent carts and smart shopping experience. A smart cart system is proposed that uses the models of smart cart devices to address the missing trolley problem using deep learning.

KEY WORDS: ABANDONED TROLLEY, MISSING TROLLEY PROBLEM, Q-LEARNING, SMART CART SOLUTION, SMART SHOPPING CART, YOLO.

\section{INTRODUCTION}

Supermarkets and shopping malls have aisles of items on display for the customers to have a look first and then buy, that is, offers self-service to the shopping experience. A shopping cart or trolley offers the customers the ease to shop and carries the items to be bought until the billing counters. The role of a trolley is not limited to the floor space of a supermarket. Many supermarkets have associated parking lots nearby. This way customers can park their vehicles while shopping. The customers use trolleys to transport the bought items from the supermarket to their vehicles in the parking lots. Artificial intelligence (AI), the Internet of Things (IoT), and other technologies have allowed for innovation in several aspects of shopping from the smart shopping experience

\section{ARTICLE INFORMATION}

*Corresponding Author: jimcymol.james@manipaldubai.com Received 11th Oct 2020 Accepted after revision 29th Dec 2020 Print ISSN: 0974-6455 Online ISSN: 2321-4007 CODEN: BBRCBA

Thomson Reuters ISI Web of Science Clarivate Analytics USA and Crossref Indexed Journal

\section{Clarivate
Analytics}

NAAS Journal Score 2020 (4.31)

A Society of Science and Nature Publication,

Bhopal India 2020. All rights reserved.

Online Contents Available at: http//www.bbrc.in/

Doi: http://dx.doi.org/10.21786/bbrc/13.13/36 to smart carts. We are focusing on exploring the features of smart trolleys.

There is also the problem of the attitudes in customers where they abandon the trolleys after use. These trolleys that end up causing nuisances for many has not been addressed in any papers. The main purpose was to propose a smart cart solution for the missing trolley problem. The proposal uses deep learning algorithms. We are also proposing a smart application for the managers to monitor the trolley usage across the customers and for the purpose of real-time location tracking.

\section{Related Work}

2.1 Use of tags on trolleys: Traditional trolleys and supermarkets are evolved into a smart shopping experience with a centralized and automated billing system (Yewatkar et al., 2016; Ramakrishnan et al., 2018). This can help in reducing the time spent in long billing queues no matter what the quantity of your cart is. This is enabled by radio frequency identification (RFID) and ZigBee modules in the build of smart carts Yewatkar et al., 2016; Ramakrishnan et al., 2018). RFID tags are used at the exit for the anti-theft property. Each trolley also has a unique Product Identification Device (PID) tag. Weight 
sensors are installed to weigh the items, making it easier to remove an item from the cart weight and subtract the difference from the total bill (Ramakrishnan et al., 2018; Gowtham et al., 2019). This is later adopted to RFID and Arduino based billing system that is automatic is implemented in reference (Rao and Madhuri, 2018). Ultra-High Frequency (UHF) RFID is used to make sure that the items in one cart are not read by the other cart by setting a reading range is proposed (Gowtham et al., 2019).

2.2 In-store Recommendation systems: In references (Yewatkar et al., 2016; Ramakrishnan et al., 2018; Gowtham et al., 2019) they also supply recommendation systems and information about the items on aisles, instore discounts, expiry dates, and many more. This is termed as Context-Aware Shopping Trolley and Personal Shopping Experience (Hosbond and Skov, 2007) were in the promoted products, shopping list, and more information can be are curated and accessed from an interactive screen mounted on the trolley (Hosbond and Skov, 2007). For example, see Fig. 1 which was designed for our project Buddy Carrello (smart cart project) showing the LCD Display getting updated with each item added or removed from the cart.

Figure 1: LCD screen designed for Buddy Carrello project

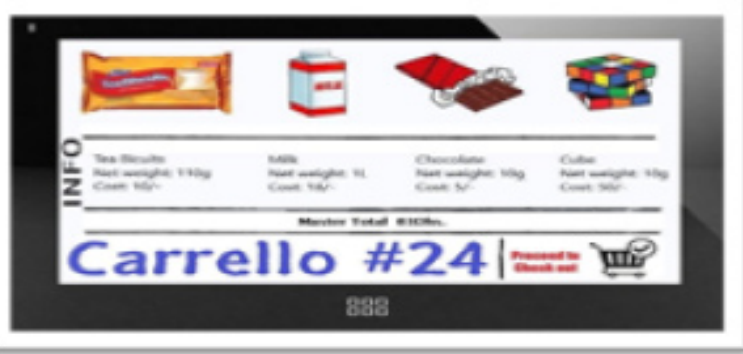

2.3 Security: Another major aspect they investigated is that when an item is removed from the shelf, the inventory needs to be updated, RFID modules are placed on the shelves for this purpose, theft precautions are also included in this paper, thereby making a complete automated shopping system (Gowtham et al., 2019). Zigbee network is used for navigation. Some models also have incorporated a lightweight cryptographic method for sending the information to the server (Puranik and Mahalle, 2018). A model used Gossamer protocol to protect the system and overcome the insecurity of the RFID tag system. They mentioned the drawbacks of the proposed system - high initial installation cost as RFID tags need to be installed on items, in shelves, and register the tags securely. Though this drawback is seen, the maintenance cost is expected to be lower.

Gap Analysis: The models and systems referred to in the literature were to address the incorporation of technologies to make the shopping carts smart when it comes to shopping. However, mostly the experience is not limited to the floorspace of the shopping malls and supermarkets. Many customers place their heavy shopping bags, push them towards their vehicles parked in the nearby parking lots and unload them into the cars. Some customers remember to safely push them out into designated parking spots for trolleys, some leave the trolleys stranded and some push the trolleys further out of the parking space and abandon them. Many trolleys are abandoned; however, no model is looking into the technical solution for the problem.

Abandoned trolleys can cause a lot problems ranging from economic losses, time losses, accidents to environmental problems. Many solutions are in-use in stores today (Bhat, 2019; Clarke and Abdullah, 2018; Barthélemy, 2020; Blaha, 2014). Some of the practices in stores to retrieve abandoned trolleys are summarized below:

- Physical systems- unlocking by insertion of coins, employee following customers, restriction on trolley movement outside the space.

- Retrieval workforce- a team of employees to retrieve the trolleys off the property of supermarket.

- $\quad$ Online and smart systems- a WhatsApp contact (as a toll-free version) to send the locations of abandons trolleys if found by customers and such online trackers, object detection through CCTV.

- Technical systems - electronic locks restricting the movement outside the property, geofencing.

- Campaigns, policies, and rewards

- However, these solutions are for traditional trolleys. A smart trolley instrumenting so many IoT devices is too precious to be replaced and need a solution to prevent any problems that exist with traditional trolleys.

Figure 2: A 3D model of smart shopping cart

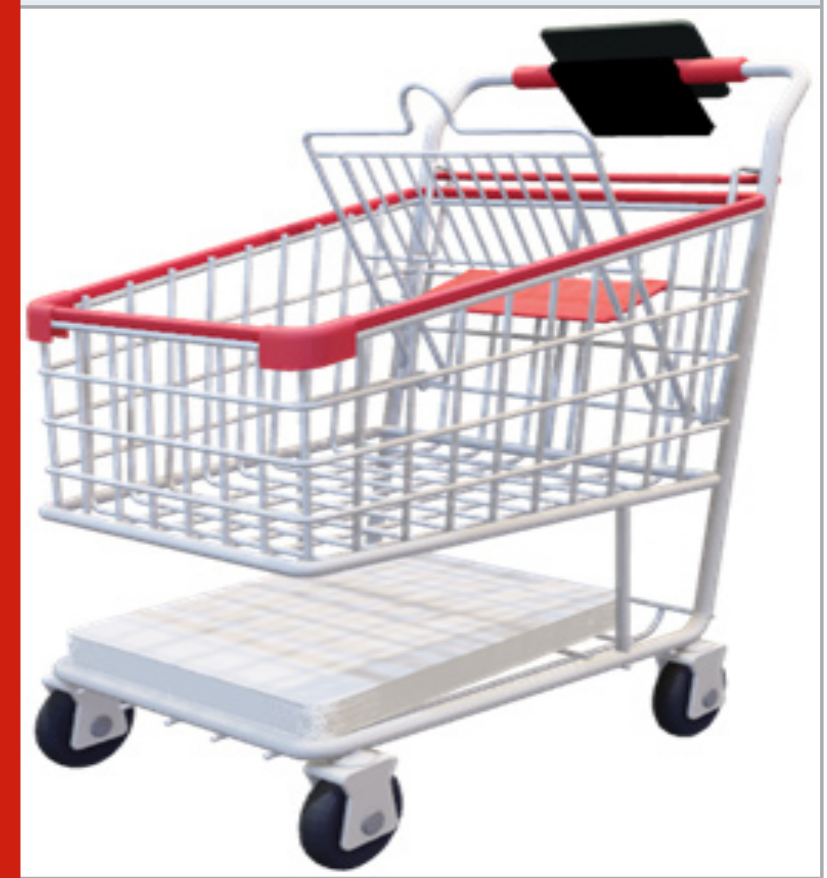


Proposed Solution: Smart shopping carts or trolleys has few devices like RFID tags, GPS locators, camera, and a display screen to provide the smart functionality. Many supermarkets already have the prototypes running. However, as previously stated there is the problem of abandoned trolleys even in a traditional supermarket scenario. Smart shopping carts or trolleys has few devices like RFID tags, GPS locators, camera, and a display screen to provide the smart functionality. Many supermarkets already have the prototypes running. However, as previously stated there is the problem of abandoned trolleys even in a traditional supermarket scenario.

Figure 3: A 3D model of smart shopping cart back to recharging station

Figure 4: Use case of Manager Application

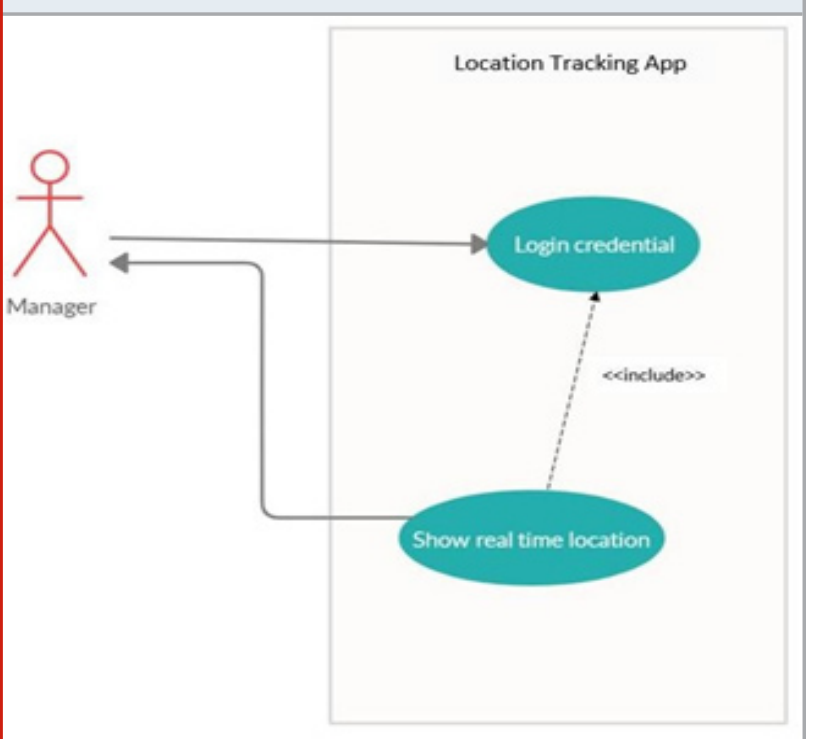

1.1. Smart cart solution: A smart trolley can use a GPS locator and signals to find the current location. Instead of a retrieval team, the trolley supplemented with autonomy will find and plan a path after being stranded in a known or unknown environment with incoming, dynamic obstacles and unpredictable perceptions (Chen et al., 2019). The best approach is Q-learning which employs the deep reinforcement learning approach. The trolley will be autonomous once it is stranded in a known or unknown environment. A Q-learning approach was experimentally found to be better performing than $\mathrm{A}^{*}$ algorithm and rapidly exploring random tree (RRT) algorithm with the increase in the number of obstacles (Chen et al., 2019).

This idea is further extended to provide the feature of autonomy inside the supermarket as well. The advantage is the addressal to a more inclusive shopping experience. A shopping mall will have various users ranging from old aged to disabled people who find it difficult to enjoy and fully experience the fun of shopping and the activity itself. The shopping cart uses You Only Look Once (YOLO) algorithm to find items on the shopping list which can be conveyed to the shoppers in a suitable manner, for example audio feedback to visually impaired shoppers. It is one of the most effective object detection algorithms under the umbrella of computer vision attributing a high accuracy and real-time working ability. YOLO a clever conversional neural network (CNN) regression algorithm that can predict by drawing multiple bounding boxes and class probabilities for those boxes. It works faster (Redmon and Farhadi, 2018) and gets better with time.

\section{Figure 5: Sample Manager application interface}

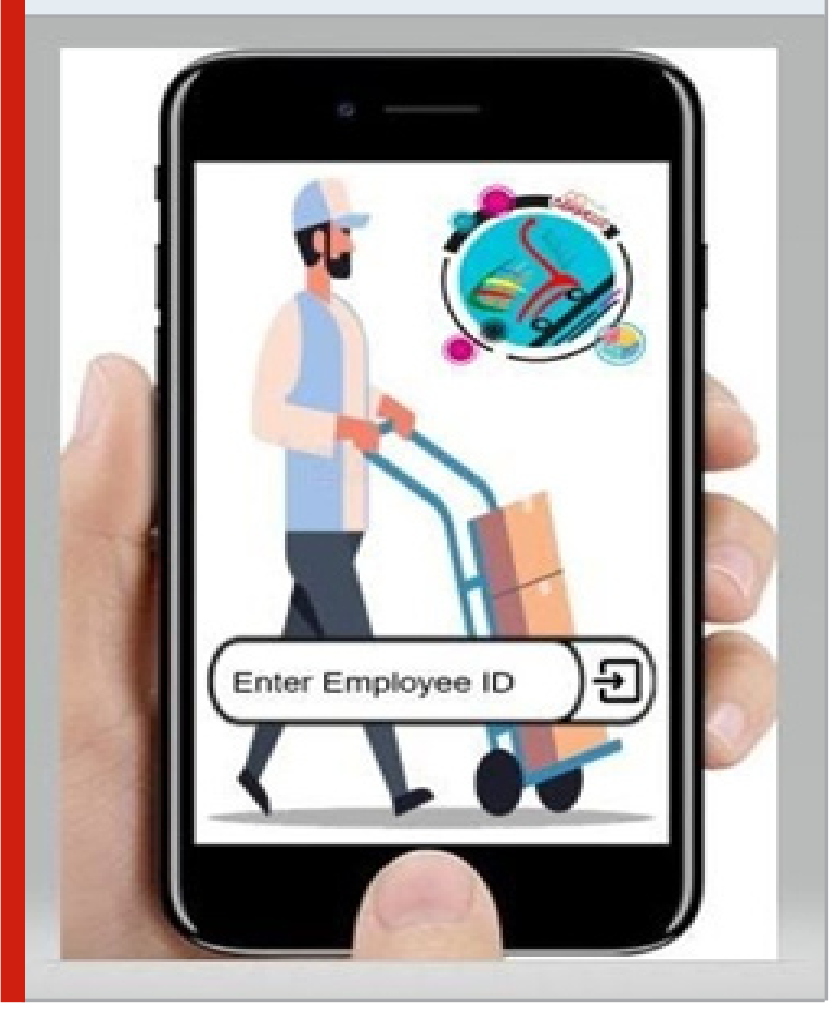

YOLO algorithms first examine the entire image captured, then boxes are drawn to generate predictions. The boxes are then used to localize, analyze, and classify the objects which is found in references (Redmon et al., 2016; Redmon and Farhadi, 2017; Redmon and Farhadi, 2018). Region based CNN (R-CNN) and faster R-CNN performs in multiple pipelines and trains each individual part separately, while YOLO works with one neural network to run all the components. YOLO makes less than half 
the number of errors than the fast R-CNN and adapts well on new domains. This makes it easier for the shops and supermarkets to update and coordinate the inventory and the server with the items.

1.2. Manager application: The proposed application is for managers to monitor, control, and locate the trolleys. This application will help in-

- identifying the current location of the missing trolleys,

- locate the current movements with the premises of the supermarkets.

- data can used be further used to understand the patterns of customer shopping with the premises.

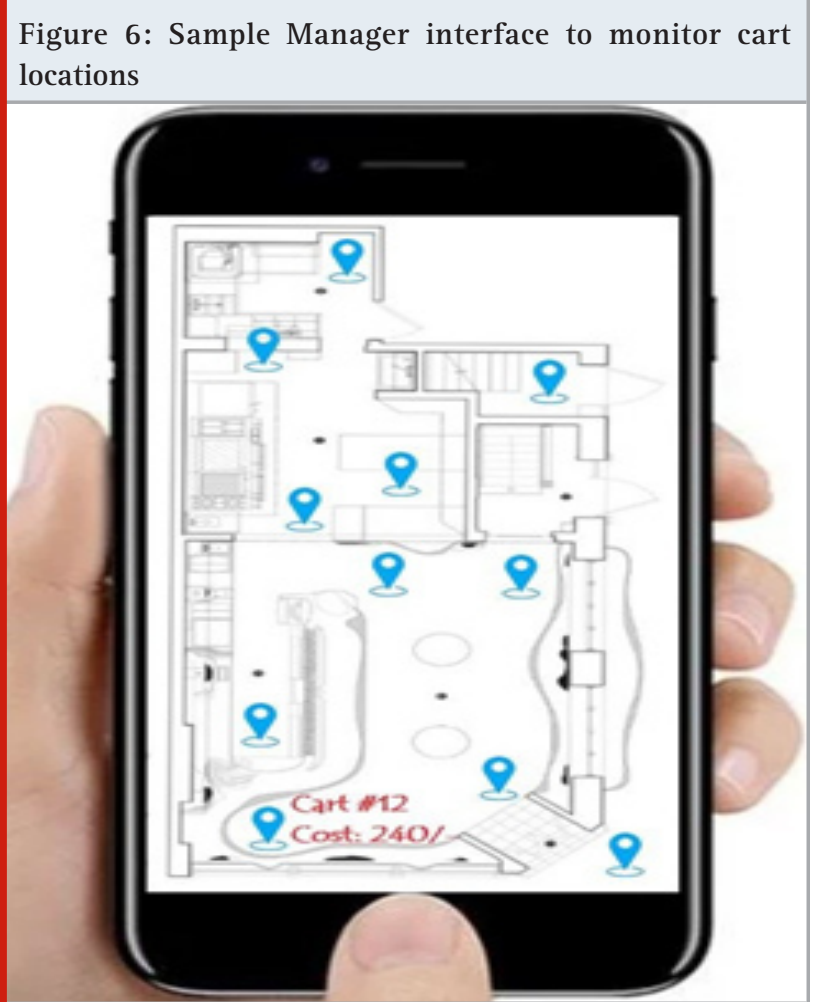

\section{RESULTS AND DISCUSSION}

RFID tags can be used by the customers to find the details of a particular item as well as in the identification of the trolleys by managers. GPS locators help in figuring out the trolley's location and movements. The positioning of the trolley is important as it determines where it is at a particular time. It can trace other factors like the speed at which it is going and the track which can help us analyze the routes for future works. With the recent forms of GPS, much better measurements can be taken, and a unique address be specified to every square meter in the mall and supermarkets.

The deep learning techniques - Q-Learning and YOLO algorithms -helps in finding the optimal path for the trolleys, allowing the trolleys to decide and to detect any incoming obstructions. YOLO processes captured images at about 45 frames per second. It also understands generalized objects, so, therefore, using the above hardware and software's this trolley will indeed be smart and never left abandoned. This smart trolley which uses deep learning techniques stands out from the other smart trolleys. Deep learning Algorithms learn and help the computer to process data very much as humans do in a structure therefore it can prevent unnecessary accidents from taking place while left abandoned and this trolley is robust as well.

\section{CONCLUSION}

We identified that the missing or abandoned trolley problem of traditional trolleys has not yet been addressed. A smart solution has been proposed in the paper using deep learning techniques for path planning and object identification. The solution proposed can be implemented in both traditional and smart shopping settings.

\section{REFERENCES}

Bhat A. (2019) Hail the supermarket trolley [Internet]. The Hindu, Available from: https://www.thehindu. com/opinion/open-page/hail-the-supermarket-trolley/ article26101293.ece.

Barthélemy, J. (2020) The war on abandoned trolleys can be won. Here's how. The Conversation [Internet]. 2020. Available from: https://theconversation.com/ the-war-on-abandoned-trolleys-can-be-won-hereshow-127718.

Blaha, M. (2014) Shopping carts turn up in the oddest of places. CNN [Internet]. Available from: https://edition. cnn.com/2014/02/24/living/irpt-missing-shoppingcarts/index.html

Clarke, K. and Abdullah, A. (2018) Missing trolleys cost big to supermarkets in UAE. Khaleej Times [Internet]. Available from: https://www.khaleejtimes.com/news/ general/missing-trolleys-cost-big-to-supermarkets-inuae.

Chen, C., Chen, X.Q., Ma, F., Zeng, X.J. and Wang, J. (2019) A knowledge-free path planning approach for smart ships based on reinforcement learning. Ocean Engineering, 189:106299. doi:10.1016/j. oceaneng.2019.106299.

Gowtham, N., Ramachandra, G. and Narasimha K. (2019) IOT APPLICATIONS ON SECURE SMART SHOPPING SYSTEM. Indian Journal of Scientific Research, 17:36070.

Hosbond, J.H. and Skov, M.B. (2007) Micro mobility marketing: Two cases on location-based supermarket shopping trolleys. Journal of Targeting, Measurement and Analysis for Marketing, 16: 68-77. doi:10.1057/ palgrave.jt.5750058.

Heng, M. (2016) Supermarkets counting cost of replacing unreturned trolleys. The New Paper [Internet], Available from: https://www.tnp.sg/news/singapore/ 
supermarkets-counting-cost-replacing-unreturnedtrolleys.

Kumar, A., Gupta, A., Balamurugan, S., Balaji, S. and Marimuthu R. (2017) Smart Shopping Cart. International conference on Microelectronic Devices, Circuits and Systems (ICMDCS), 2017:1-4, doi: 10.1109/ ICMDCS.2017.8211723.

Karjol, S., Holla, A.K. and Abhilash, C.B. (2018) An IOT Based Smart Shopping Cart for Smart Shopping. In:

Li, R., Song, T., Capurso, N., Yu, J., Couture, J. and Cheng, X. (2017) IoT Applications on Secure Smart Shopping System. In IEEE Internet of Things Journal, 4(6):1945-1954. doi: 10.1109/JIOT.2017.2706698.

Nagabhushan, T., Aradhya, V., Jagadeesh, P., Shukla S. (2017) Cognitive Computing and Information Processing. Communications in Computer and Information Science, 801:978-981. https://doi.org/10.1007/978-981-10-90592_33

Puranik, P.S. and Mahalle, P.N. (2018) IoT Application on Smart and Secure Shopping System using RFID, Zig-Bee and Gossamer Protocol. International Journal of Engineering and Techniques, 4:374-9.

Ramakrishnan, D., Pavithra, T., Monisha, E. and Likitha, M. (2018) IoT APPLICATIONS ON SECURE SMART SHOPPING SYSTEM. International Research Journal of Engineering and Technology (IRJET), 5: 2395-0056. Rao, K.S. and Madhuri, C. (2018) National Conference on Emerging trends in Information, management and Engineering sciences "NC'ea-TIMES\# 1.0"-2018. International Journal of Engineering Technology.

Redmon, J., Divvala, S., Girshick, R. and Farhadi, A. (2016) You Only Look Once: Unified, Real-Time Object Detection. IEEE Conference on Computer Vision and Pattern Recognition (CVPR), doi:10.1109/cvpr.2016.91. Redmon, J. and Farhadi, A. (2017) YOL09000: Better, Faster, Stronger. IEEE Conference on Computer Vision and Pattern Recognition (CVPR), doi:10.1109/ cvpr.2017.690.

Redmon, J and Farhadi A. (2018) YOLOv3: An Incremental Improvement.

Shahroz, M., Mushtaq, M.F., Ahmad, M., Ullah, S., Mehmood, A. and Choi G. S. (2020) IoT-Based Smart Shopping Cart Using Radio Frequency Identification. IEEE Access, 8:68426-68438. doi: 10.1109/ACCESS.2020.2986681.

Viswanadha, V., Pavan Kumar, P. and Chiranjeevi Reddy, S. (2018) Smart Shopping Cart. International Conference on Circuits and Systems in Digital Enterprise Technology (ICCSDET, 1-4, doi: 10.1109/ICCSDET.2018.8821103. Yewatkar, A., Inamdar, F., Singh, R. and Bandal, A. (2016) Smart Cart with Automatic Billing, Product Information, Product Recommendation Using RFID Etamp; Zigbee with Anti-Theft. Procedia Computer Science, 79:793800. doi: 10.1016/j.procs.2016.03.107. 